\title{
Benchmarking Monte Carlo Codes for Criticality Safety Using Subcritical Measurements
}

\author{
T. Valentine \\ Oak Ridge National Laboratory, USA \\ P. O. Box 2008 Oak Ridge, TN 37831 USA \\ Phone: (865) 574-6176 \\ Fax: (865) 574-6182 \\ email: valentinete@ornl.gov
}

\begin{abstract}
Monte Carlo codes that are used for criticality safety evaluations are typically validated using critical experiments in which the neutron multiplication factor is unity. However, the conditions for most fissile material operations do not coincide to those of the critical experiments. This paper demonstrates that Monte Carlo methods and nuclear data can be validated using subcritical measurements whose conditions may coinicide more closely to actual configurations of fissile material.
\end{abstract}

\section{Introduction}

The subcriticality of fissile assemblies is essential for safe processing, transportation, and storage operations. The assurance of the subcriticality of various fissile material operations is commonly obtained by criticality safety evaluations for the specific operation. The criticality safety evaluations are performed using detailed computations that have been validated using critical experiments in which the neutron multiplication factor is unity. In most cases, the actual fissile material operation does not coincide to the conditions that exist for the critical experiment, i.e. the geometry and neutron spectrum may both differ significantly between the subcritical operation and the critical experiment. These differences require that additional safety margins be included in the criticality safety evaluations [1]. Subcritical measurements can be used to address the use of additional safety margins by providing supplementary benchmark data for validating computation methods or by providing an in-situ measurement of the degree of subcriticality. The use of subcritical measurements would actually enhance the safety of operations with fissile materials.

This paper provides a brief review of subcritical noise analysis measurements followed by a description of the simulation of this measurement method. A brief description of a noise analysis measurement and the results of the simulation of this measurement are provided. Finally, the results of the analysis are summarized.

\section{Source-Driven Noise Analysis Measurements}

Subcritical source-driven noise measurements [2] are simultaneous Rossi- $\alpha$ and randomly pulsed neutron measurements that provide measured quantities that 
can be related to the subcritical neutron multiplication factor, $k_{e f f}$. The sourcedriven noise analysis measurement requires the use of a timed neutron source such as a ${ }^{252} \mathrm{Cf}$ source ionization chamber (detector 1) and two or more neutron detectors (detectors 2 and 3, respectively). The time-dependent response of the source and detectors are correlated with themselves and each other in the frequency domain to obtain auto and cross spectra. The source-detector cross spectra are designated as $G_{12}(\omega)$ and $G_{13}(\omega)$ and are the frequency domain equivalent of the randomly pulsed neutron measurement. The detector-detector cross sepctra are designated as $G_{23}(\omega)$ and are the frequency domain equivalent of the two-detector Rossi- $\alpha$ measurement. The auto spectra of the source is designated as $G_{11}(\omega)$ and is related to the spontaneous fission rate of the source. The detector auto spectra are designated as $G_{22}(\omega)$ and $G_{33}(\omega)$ and are the frequency domain equivalent to the single-detector Rossi- $\alpha$ measurement. A certain ratio $(R)$ of the frequency spectra is independent of detection efficiency and can be directly computed using Monte Carlo codes. The spectral ratio is defined as:

$$
R(\omega)=\left(G_{12}^{*}(\omega) G_{13}(\omega)\right) /\left(G_{11}(\omega) G_{23}(\omega)\right)
$$

The spectral ratio is directly dependent on the subcritical neutron multiplication factor and thus can be used to acertain changes in reactivity.

\section{Monte Carlo Code MCNP-DSP}

Subcritical measurements were developed to study the dynamic behavior of neutrons in a fissile system. One common aspect of this was to determine the neutron multiplication factor. Interpretation of measurements requires some model to relate what is measured to the neutron multiplication factor. In the past, the subcritical measurements were interpreted using equations developed from point kinetics models for the time-dependent behavior of neutrons in the subcritical configuration. This limited the application of the measurement to situations in which point kinetics was applicable. The most general model to relate the measured quantities to keff would involve the use of the generalized stochastic model developed by Munoz-Cobos et al [3]. Although an analytical solution of subcritical measurements in terms of the stochastic model is not practical, the Monte Carlo method provides a means to simulate the subcritical measurements and to also calculate the neutron multiplication factor. In fact, the same Monte Carlo code and nuclear data can be used for both calculations.

The Monte Carlo code MCNP-DSP [4] was developed from the MCNP [5] Monte Carlo code to simulate a variety of subcritical measurements. In MCNPDSP, the variance reduction features were disabled to obtain a strictly analog particle tracking to follow the fluctuating processes more accurately. Because typical biasing techniques are employed to reduce the variance of estimates of first moment quantities, they do not preserve the higher moments; therefore, analog Monte Carlo calculations must be performed when analyzing subcritical 
measurements whose measured quantities are directly related to the higher moments of the neutron production. Because the use of average quantities reduces the statistical fluctuations of the neutron population, average quantities such as the average number of neutrons from fission are not used; instead, appropriate probability distribution functions are sampled. The prompt particle tracking begins with the source event and the subsequent fission chains are followed to extinction. Time series of pulses are obtained at the detectors for each fission chain. These sequences are sampled into blocks of 512 or 1024 data points. The blocks of data are then processed according to the type of measurement being simulated. The auto and cross spectra are computed directly from the Fourier transform of the source and detector time series for each data block. The auto and cross spectra are then averaged over many blocks to obtain the final estimates of the auto and cross spectra. The spectral ratio can then be computed from the auto and cross spectra as is done in the noise analysis measurement.

\section{Comparison of Measurements and Simulations}

\subsection{Measured Spectral Ratio Values}

Source-driven noise analysis measurements were performed with varying amounts of a highly enriched uranyl nitrate solution in an acrylic tank (25.0825-cm ID, $53.34-\mathrm{cm}$ height) to obtain the spectral ratio as a function of the solution height in the tank [6]. A $0.635-\mathrm{cm}$ stainless steel plate was located at the bottom of the solution tank. A ${ }^{252} \mathrm{Cf}$ source was placed at the axial center of the tank at the mid-height of the solution. ${ }^{3} \mathrm{He}$ neutron detectors (5.08-cm OD, 38.1-cm long)were placed adjacent to the radial surface of the tank and were separated 180 degrees apart. The aqueous uranyl nitrate solution contained 0.29330 gram of uranium per gram of solution. The solution density was $1.64320 \mathrm{~g} / \mathrm{cm}^{3}$ and had a free acid content $<0.1 \mathrm{wt} \% \mathrm{HNO}_{3}$. The uranium isotopic content was 93.2 wt $\%{ }^{235} \mathrm{U}, 5.37 \mathrm{wt} \%{ }^{238} \mathrm{U}, 1.02 \mathrm{wt} \%{ }^{234} \mathrm{U}$, and $0.41 \mathrm{wt} \%{ }^{236} \mathrm{U}$. A detailed sensitivity study was performed for this measurement to determine the benchmark spectral ratio values and uncertainties that are presented in Table 1.

\subsection{MCNP-DSP Spectral Ratio Values}

MCNP-DSP calculations were performed to simulate these measurements using ENDF/B-V and ENDF/B-VI neutron cross section data files. The computed spectral ratio values and $k_{e f f}$ values are provided in Tables 2 and 3 . The statistical uncertainty in the $k_{\text {eff }}$ calculations is 0.0003 for both cross section data libraries. The spectral ratio values computed with the ENDF/B-VI cross section data set are in better agreement with the measurements for the 30.48-and 27.94$\mathrm{cm}$ solution heights and are very similar to those obtained using the ENDF/B-V cross section data for the other four solution heights. The computed spectral ratio values are in better agreement for the more reactive systems than for the 
Table 1. Benchmark Spectral Ratio Values

\begin{tabular}{cc}
\hline$H$ & $R$ \\
$(\mathrm{~cm})$ & $\left(10^{-3}\right)$ \\
\hline 30.48 & $98.0 \pm 12.0$ \\
27.94 & $139.0 \pm 9.7$ \\
25.40 & $187.7 \pm 10.6$ \\
22.86 & $248.0 \pm 9.7$ \\
20.32 & $303.5 \pm 9.7$ \\
\hline
\end{tabular}

less reactive systems. As the solution height decreases the reactivity of the system decreases and greater differences are observed between the measured and computed spectral ratio values.

The spectral ratio is a more sensitive parameter than $k_{e f f}$ as evident from the results presented in Tables 2 and 3. For example, the computed spectral ratio values change approximately $40 \%$ for a change in solution height from $30.48-\mathrm{cm}$ to $27.94-\mathrm{cm}$ whereas the computed $k_{e f f}$ values only change approximately $2 \%$. The sensitivity of the computed spectral ratio to solution height decreases as the solution height decreases whereas the sensitivity of the computed $k_{e f f}$ increases as the solution height decreases. However, the computed spectral ratio is still a factor of three more sensitive to the change in solution height than $k_{e f f}$.

Table 2. MCNP-DSP Spectral Ratio Values

\begin{tabular}{ccc}
\hline & $E N D F / B-V$ & $E N D F / B-V I$ \\
$H$ & $R$ & $R$ \\
$(\mathrm{~cm})$ & $\left(10^{-3}\right)$ & $\left(10^{-3}\right)$ \\
\hline 30.48 & $105.5 \pm 0.1$ & $102.7 \pm 0.1$ \\
27.94 & $151.3 \pm 0.1$ & $149.4 \pm 0.1$ \\
25.40 & $202.9 \pm 0.1$ & $201.6 \pm 0.2$ \\
22.86 & $259.5 \pm 0.3$ & $268.1 \pm 0.3$ \\
20.32 & $328.4 \pm 0.3$ & $327.0 \pm 0.8$ \\
\hline
\end{tabular}

\section{Summary}

These calculations have demonstrated that subcritical source-driven noise analysis measurements can be simulated directly using Monte Carlo codes. The 
Table 3. MCNP-DSP Spectral Ratio Values

\begin{tabular}{ccc}
\hline$H$ & $E N D F B-V$ & $E N D F B-V I$ \\
$(\mathrm{~cm})$ & $k_{e f f}$ & $k_{e f f}$ \\
\hline 30.48 & 0.9599 & 0.9582 \\
27.94 & 0.9391 & 0.9381 \\
25.40 & 0.9141 & 0.9137 \\
22.86 & 0.8829 & 0.8820 \\
20.32 & 0.8445 & 0.8435 \\
\hline
\end{tabular}

sensitivity of the computations has been demonstrated using ENDF/B-V and ENDF/B-VI cross section data libraries. Futhermore, these calculations have demonstrated that the bias between the measurement and calculations (difference between the measured and calculated spectral ratio values) increases as the system reactivity decreases. The bias is smaller for highly reactive systems. This result might be expected because the evaluated nuclear data is typically tested using critical experiments in which the neutron multiplication factor is one. The increased sensitivity of the spectral ratio as a function of the degree of reactivity makes this quantity useful for benchmarking Monte Carlo methods and nuclear data.

Acknowledgement This work was performed at Oak Ridge National Laboratory which is managed by UT-Battelle, LLC, under contract DE-AC05-00OR22725 with the U.S. Department of Energy.

Disclaimer-The submitted manuscript has been authored by a contractor of the U. S. Government. Accordingly, the U. S. Government retains a nonexclusive, royalty-free license to publish or reproduce the published form of this contribution, or allow others to do so, for U. S. Government purposes.

\section{References}

1. ANSI (American National Standards Institute), 1998. "Nuclear Criticality Safety in Operations with Fissionable Materials Outside Reactors," ANSI/ANS-8.1-1998, American Nuclear Society.

2. V. K. Par and J. T Mihalczo, Nucl. Sci. Eng., 56, 213 (1975).

3. 18. J. L. Munoz-Cobo, R. B. Perez, and G. Verdu, Nucl. Sci. Eng., 95, 83 (1987).

4. Valentine, T.E., MCNP-DSP Users Manual, ORNL/TM-13334, Oak Ridge Nat. Lab, January 1997.

5. Briesmeister, J. F., Ed., "MCNP4A-A General Monte Carlo N-Particle Transport Code," LA-12625-M, Los Alamos National Laboratory, 1993.

6. Mihalczo, J. T., Blakeman, E. D., Ragan, G. E., Johnson, E. B., and Hachiya, Y., "Dynamic Subcriticality Measurements Using the 252Cf-Source-Driven Noise Analysis Method," Oak Ridge National Laboratory, ORNL/TM-10122 (1988). 